\title{
Direct visualization of microcirculation impairment after acute subdural hemorrhage in a novel animal model
}

\author{
Huan-Chih Wang, MD,1,2 Jui-Chang Tsai, MD, PhD, ${ }^{2}$ Jing-Er Lee, MD, PhD, ${ }^{3}$ \\ Sheng-Jean Huang, MD, ${ }^{2}$ Abel Po-Hao Huang, MD, ${ }^{2}$ Wei-Chou Lin, MD, PhD, ${ }^{4}$ \\ Sung-Tsang Hsieh, MD, PhD, ${ }^{5,6}$ and Kuo-Chuan Wang, MD, PhD²
}

\begin{abstract}
1Division of Neurosurgery, Department of Surgery, National Taiwan University Hospital Hsinchu Branch, Hsinchu; ${ }^{2}$ Division of Neurosurgery, Department of Surgery, ${ }^{4}$ Department of Pathology, and ${ }^{5}$ Department of Neurology, National Taiwan University Hospital and National Taiwan University College of Medicine, Taipei; ${ }^{3}$ Department of Neurology, Taipei Medical University-Wan Fang Hospital, Taipei; and ${ }^{6}$ Department of Anatomy and Cell Biology, College of Medicine, National Taiwan University, Taipei, Taiwan
\end{abstract}

\begin{abstract}
OBJECTIVE Direct brain compression and secondary injury due to increased intracranial pressure are believed to be the pathognomic causes of a grave outcome in acute subdural hemorrhage (aSDH). However, ischemic damage from aSDH has received limited attention. The authors hypothesized that cerebral microcirculation is altered after aSDH. Direct visualization of microcirculation was conducted in a novel rat model.
\end{abstract}

METHODS A craniectomy was performed on each of the 18 experimental adult Wistar rats, followed by superfusion of autologous arterial blood onto the cortical surface. Changes in microcirculation were recorded by capillary videoscopy. Blood flow and the partial pressure of oxygen in the brain tissue $\left(\mathrm{P}_{\mathrm{bt}} \mathrm{O}_{2}\right)$ were measured at various depths from the cortex. The brain was then sectioned for pathological examination. The effects of aspirin pretreatment were also examined.

RESULTS Instantaneous vasospasm of small cortical arteries after aSDH was observed; thrombosis also developed 120 minutes after aSDH. Reductions in blood flow and $\mathrm{P}_{\mathrm{bt}} \mathrm{O}_{2}$ were found at depths of 2-4 mm. Blood-brain barrier disruption and thrombi formation were confirmed using immunohistochemical staining, while aspirin pretreatment reduced thrombosis and the impairment of microcirculation.

CONCLUSIONS Microcirculation impairment was demonstrated in this aSDH model. Aspirin pretreatment prevented the diffuse thrombosis of cortical and subcortical vessels after aSDH.

https://thejns.org/doi/abs/10.3171/2017.5.JNS162579

KEY WORDS acute subdural hemorrhage; microcirculation; aspirin; rat model; trauma

$\mathrm{T}$ RAUMATIC brain injury (TBI) accounts for a significant number of deaths worldwide. ${ }^{16}$ Among all types of injury, acute subdural hemorrhage (aSDH) is recognized as one of the most devastating. Previous studies have reported mortality rates of $40 \%-70 \%^{14,19,34}$ with aSDH; the rate has lowered to $14 \%-46 \%$ in recent years, ${ }^{36,42,43}$ which may be attributed to the widespread use of CT, improvement in surgical techniques, and delivery of specialized care within trauma centers. However, this improved mortality rate, in combination with the morbidity rate, is still frustratingly high. ${ }^{23,36}$ The mechanism of injury is believed to be a direct compression effect caused by aSDH and an indirect effect or secondary brain injury caused by increased intracranial pressure (ICP). Ischemic damage might be another cause, but this has not been investigated in such detail. The possible mechanisms of hematoma-related brain damage have been extensively discussed in cases of intracerebral hemorrhage (ICH) ${ }^{31,32}$ and subarachnoid hemorrhage (SAH), ${ }^{30,41,44}$ but have seldom been investigated in cases of aSDH. Based on the finding that ischemic brain damage was noted in humans who died after aSDH, ${ }^{2}$ Baechli et al. ${ }^{4}$ proposed that is-

ABBREVIATIONS aSDH = acute subdural hemorrhage; BBB = blood-brain barrier; CCD = charge-coupled device; GLUT1 = glucose transporter 1 ; ICH = intracerebral hemorrhage; $I C P=$ intracranial pressure; $I H C=$ immunohistochemical; $I P=$ intraperitoneal; $P B S=$ phosphate-buffered saline; $P_{b t} O_{2}=$ partial pressure of oxygen in the brain tissue; RBC = red blood cell; SAH = subarachnoid hemorrhage; SMA = smooth muscle actin; SVZ = subventricular zone; TBI = traumatic brain injury.

SUBMITTED October 16, 2016. ACCEPTED May 23, 2017.

INCLUDE WHEN CITING Published online December 8, 2017; DOI: 10.3171/2017.5.JNS162579. 
chemia triggered by blood constituents may worsen the outcome of aSDH. Other studies have attempted to investigate the influence of blood constituent-induced ischemia on the outcome of aSDH, but no definite conclusion was reached. ${ }^{12,20,22}$ In the clinical setting, we have observed that the density of the cortical vasculature is evidently smaller in patients with aSDH under microscopic view (therefore implying impairment of microcirculation), and it is not increased after dural opening and hematoma evacuation; this condition can be improved significantly after normal saline irrigation (case examples below).

In Case 1, a 78-year-old female patient presented to the emergency department with sudden-onset slurred and disorganized speech after a fall at home. The patient's vital signs were normal, and no physical abnormality was evident. Neurological examination showed expressive aphasia, with symmetric muscle power and reactive pupils. Initial laboratory investigations showed microcytic anemia and marked thrombocytopenia, and further brain CT showed left frontotemporal aSDH and a mild midline shift. Conservative treatment was provided at first, but several partial seizure episodes occurred within 4 hours of hospitalization, despite early administration of anticonvulsant medications. The patient became drowsy, and a right hemiparesis was also noted. A left frontotemporal craniotomy was then performed to evacuate the aSDH, and an obvious difference in the density of the cortical vasculature was noted after vigorous saline irrigation of the subdural space (Fig. 1A-C). Some of the necrotic area did not recover well from the irrigation. Persistent partial seizures lasted for 5 more days after the surgery under aggressive medical management. The subsequent course of hospitalization was complicated with acute kidney injury and septic shock, and the patient died 1 month after the surgery.

In Case 2, a 52-year-old female patient presented to the emergency department with a head injury after a motor vehicle accident. Her consciousness was clear, and no focal neurological deficit was present initially. Repeated partial seizures of the limbs on the right side occurred in the following days. Brain CT showed a thin left-sided aSDH and a mild midline shift. Due to poor control of the focal seizures and progressive drowsiness, a left frontotemporal craniotomy was performed 2 days after the initial impact to evacuate the aSDH. The density of the cortical vasculature was noted to be poor after dural opening, and remained poor after removal of the hematoma. We also observed that the venous drainage had collapsed, but significantly improved after irrigation with large amounts of saline (Fig. 1D-F). The patient recovered smoothly with no obvious neurological deficit after the surgery and during the subsequent outpatient follow-up.

Both of these cases demonstrated disproportionate neurological deficits in relation to the thin layer of aSDH (which is usually managed conservatively). Intraoperative findings of a decreased cortical vasculature density with partial reversibility after saline irrigation implied vasoconstriction and impaired microcirculation. Because the vasoconstriction did not recover immediately after aSDH removal, but did improve after saline irrigation (which also removed the hematoma metabolites), this observation urged us to investigate the impairment of cerebral micro- circulation resulting from aSDH under compression-free conditions.

The aim of this study was to investigate the impairment of microcirculation after aSDH via direct visualization of microcirculation using a novel rat model. Animal models of aSDH previously proposed were developed by injecting blood into the subdural space, resulting in increased ICP. ${ }^{18,37}$ To test our hypothesis that altered cerebral microcirculation can be caused by the blood itself and its subsequent metabolites, rather than by the compressive effect of the aSDH, our model eliminated the pressure effect by craniectomy and durotomy. Blood without anticoagulant was then introduced onto the brain surface, therefore leaving the brain with a layer of aSDH uncompressed in an open space. The effects of aSDH on the cerebral microcirculation could then be observed both structurally and pathologically by direct observation of the vasculature alterations and measurements of regional blood flow and tissue oxygenation. The possible protective effect of aspirin was also examined in this model.

\section{Methods \\ General Preparations}

This study was approved by the National Taiwan University Hospital Committee of Animal Research and conducted in compliance with animal ethics regulations. A total of 18 Wistar rats, weighing 250-300 g, were used for our experiments. Six rats each were randomized into the control group, the aSDH without aspirin pretreatment group, and the aSDH with intraperitoneal (IP) aspirin pretreatment group. The animals were anesthetized with $1.5 \%$ halothane in a mixture of $30 \% \mathrm{O}_{2} / 70 \% \mathrm{~N}_{2} \mathrm{O}$. A prone position was adopted, and a sagittal scalp incision was made in each rate. A craniectomy window measuring $5 \times 5 \mathrm{~mm}$ on the brain convexity, behind the coronal suture and $2 \mathrm{~mm}$ off the midline, was created using a saline-cooled drill. The dura mater was opened and meticulously checked for bleeding with gentle gauze packing, eliminating any significant compression of the brain cortex. The right femoral artery was cannulated for continuous arterial blood pressure monitoring using an RFT biomonitor (VEB Messgeraetewerk). Body temperature was maintained at $37^{\circ} \mathrm{C}$ $\pm 0.5^{\circ} \mathrm{C}$ using a heating pad. The depth of anesthesia was monitored by measuring blood pressure (continuous arterial pressure recording from the right femoral artery) and response to tail pinching.

\section{Induction of SDH}

A total of $0.3 \mathrm{ml}$ of blood was drawn from the right femoral artery without an anticoagulant. To eliminate the pressure effect caused by injecting the blood into a closed subdural space, we superfused the blood gently onto the exposed subdural space (with the overlying dura and skull removed) over a 5-minute period with the animal subject placed in a flat head-down position. Precaution was taken to maintain full coverage of the exposed brain surface with superfused blood.

\section{Recording of the Cortical Vasculature}

A CAM1 capillary anemometer (KK Technology) with 

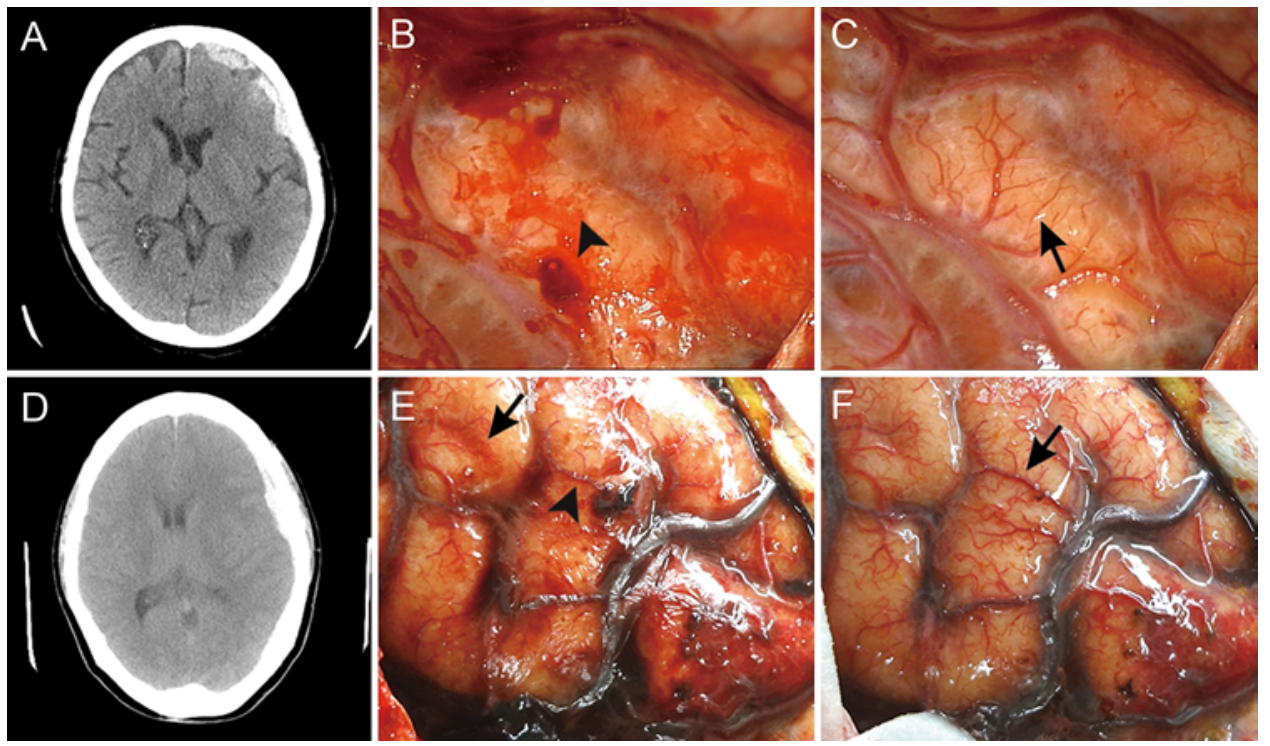

FIG. 1. Images from 2 cases of microcirculation impairment after aSDH. Case 1 (A-C). A 78-year-old female patient sustained a head injury from a fall and presented with expressive aphasia and repeated seizures. A: Axial brain CT demonstrated a small left frontotemporal aSDH with only a mild midline shift. B: Intraoperative photography showed decreased cortical vasculature and an area of necrosis (arrowhead). C: After vigorous saline irrigation, the density of the vasculature increased (arrow). Case 2. (D-F) A 52-year-old female patient sustained a head injury in a motor vehicle accident. Repeated seizures occurred after the patient's arrival at the hospital. D: Axial brain CT demonstrated a thin left-sided aSDH and a mild midline shift. E: Intraoperative photography showed a necrotic area (arrow) and diminishing cortical microvasculature, accompanied by collapse of the cortical vein (arrowhead). F: Hyperemia of the cortical microvasculature and inflation of the cortical vein (arrow) were noted after vigorous saline irrigation. Figure is available in color online only.

a high-resolution $(752 \times 582$ pixel $)$ monochrome chargecoupled device (CCD) video camera was used. The field of vision was $684 \times 437 \mu \mathrm{m}$, and the image was magnified to give an overall magnification of approximately $0.91 \mu \mathrm{m} /$ pixel. A microscope was attached to a heavy support to allow 3D adaptation without contact with the brain surface. The cortical vasculature was recorded using capillary videoscopy at fixed time points $(0,5,30,60$, and 120 minutes after blood superfusion).

\section{Measurement of Regional Brain Blood Flow and $\mathrm{P}_{\mathrm{bt}} \mathrm{O}_{2}$}

Tissue perfusion (regional brain blood flow) and the partial pressure of oxygen in the brain tissue $\left(\mathrm{P}_{\mathrm{bt}} \mathrm{O}_{2}\right)$ of the brain cortex were measured using OxyLite 2000E and OxyFLO 2000E detectors (Oxford Optronic Ltd). The detection probe was initially placed at $2 \mathrm{~mm}$ in depth from the brain surface. After this initial recording, the probe was gradually advanced until it reached $7 \mathrm{~mm}$ in depth, with continuous recording of the perfusion parameters.

\section{Brain Preparation for Immunohistochemical Analysis}

Rats were killed 4 hours after SDH by a $200 \mathrm{mg} / \mathrm{kg}$ IP injection of pentobarbital and perfused transcardially with $50 \mathrm{ml}$ of saline followed by $500 \mathrm{ml}$ of a fixative containing $4 \%$ paraformaldehyde in $0.1 \mathrm{M}$ phosphate-buffered saline (PBS) at $\mathrm{pH} 7.3$ for 30 minutes. Serial coronal brain sections (6-mm thick) were cut on a cryostat, thaw-mounted onto gelatin-coated slides, and subjected to immunohistochemical (IHC) staining. The samples were deparaffinized by heating at $60^{\circ} \mathrm{C}$ for 30 minutes and applying xylene, then rehydrated by passing through a series of decreasing concentrations of ethanol $(100 \%, 90 \%, 70 \%$, and $50 \%$ ) for 5 minutes per step, and washed with 0.1 M PBS. Endogenous peroxidase was quenched with $3 \%$ hydrogen peroxidase for 10 minutes.

After preparation, $\mathrm{H} \& \mathrm{E}$ staining, Evans blue staining, and IHC staining targeting albumin were performed as the initial steps of pathological examination. IHC staining with fluorescent dyes including fibrin (green in fluorescence), glucose transporter 1 (GLUT1; red in fluorescence), and $\alpha$-smooth muscle actin (SMA; also red in fluorescence) was then performed. Single immunofluorescence staining for GLUT1 was performed for morphological analysis of the cortical and subcortical vessels. Rat brain sections were incubated with primary antibody (GLUT1 1:200, ab652, Abcam) at $4^{\circ} \mathrm{C}$ overnight, and the secondary antibody, Alexa Fluor 594 (1:500, goat antirabbit immunoglobulin G, Invitrogen, Molecular Probes), was used at room temperature for 30 minutes. Each immunofluorescence-stained section was digitized (at $3 \times 2$ $\mathrm{mm}^{2}$ ) for measurement of the area of GLUT1 immunoreactive vessels and the area of autofluorescent blood cells using a 3-CCD color video camera (Zeiss Axiovert 200 M) interfaced with the MetaMorph image analysis system. The thrombotic percentage was calculated as the area of blood cells divided by GLUT1.

\section{Pretreatment With Aspirin}

Intraperitoneal injection of aspirin solution $(150 \mathrm{mg} /$ $\mathrm{kg}$ ) was performed 1 hour before craniotomy in the aSDH 
with aspirin pretreatment group. The general preparation and the subsequent measurements were otherwise identical to those of the aSDH without aspirin pretreatment group.

\section{Statistical Analysis}

Statistical analyses were performed using the statistical program SAS (version 9.1.3, SAS Institute Inc.) and PASW Statistics (version 18, SPSS Inc.). The data were subjected to 1-way ANOVA, followed by Tukey's post hoc test for comparisons among groups. All values were expressed as means with the standard error of the mean. A p value of less than 0.05 was regarded as significant.

\section{Results}

\section{Impairment of Microcirculation After Induction of aSDH}

A baseline picture of the cortical vasculature was first acquired for subsequent comparison. Thrombosis developed and gradually proceeded to complete obliteration of all of the cortical terminal arterioles. Laser duplex scanning aimed at these arterioles failed to detect any flow, and the flow velocity also significantly reduced in the venules. Disaggregation of red blood cells (RBCs) was clearly observed, which indicated a decreased flow velocity. The RBCs then became static in place, and the venules ultimately became thrombosed 120 minutes after the induction of aSDH (Fig. 2, Video 1).

VIDEO 1. Clip showing that as time elapsed, stasis of blood flow became evident at 120 minutes after induction of aSDH. Copyright

Kuo-Chuan Wang. Published with permission. Click here to view.

The thrombotic vessels were resistant to normal saline irrigation (Fig. 2E and I). A gross picture of the brain surface after transcardiac perfusion with normal saline 120 minutes after aSDH showed diffuse thrombosis of cortical vessels and distinct areas of necrosis (Fig. 2F).

\section{Significant Reduction of Regional Blood Flow and $\mathrm{P}_{\mathrm{bt}} \mathrm{O}_{2}$}

The arterial blood pressure, continuously measured from the right femoral artery, did not differ during the entire recording duration across all groups, indicating that the same anesthetic depth and circulatory status were maintained. Regional blood flow and $\mathrm{P}_{\mathrm{bt}} \mathrm{O}_{2}$ values recorded at a depth of $2 \mathrm{~mm}$ from the cortex showed rapid reduction of regional blood flow and $\mathrm{P}_{\mathrm{bt}} \mathrm{O}_{2}$ after induction of aSDH. The decrease in regional blood flow became significant 10 minutes after aSDH (aSDH vs control, $\mathrm{p}<$ 0.01 at a depth of $2 \mathrm{~mm}$ ), whereas the differences in $\mathrm{P}_{\mathrm{bt}} \mathrm{O}_{2}$ reached statistical significance after the 90 -minute recording period (aSDH vs control, $\mathrm{p}<0.01$ at a depth of $2 \mathrm{~mm}$; Fig. 3A-C).

Two hours after aSDH, blood flow and $\mathrm{P}_{\mathrm{bt}} \mathrm{O}_{2}$ values recorded at variable depths under the brain surface (from 2 $\mathrm{mm}$ to $7 \mathrm{~mm}$, at 1 -mm intervals) were significantly different only at certain depths from the surface. Significant reductions in blood flow (aSDH vs control, $\mathrm{p}<0.01$ at 2 $\mathrm{mm}$; aSDH vs control, $\mathrm{p}<0.01$ at $3 \mathrm{~mm}$ ) and $\mathrm{P}_{\mathrm{bt}} \mathrm{O}_{2}$ (aSDH vs control, $\mathrm{p}<0.01$ at $2 \mathrm{~mm}$; aSDH vs control, $\mathrm{p}<0.01$ at $3 \mathrm{~mm}$; aSDH vs control, p $<0.01$ at $4 \mathrm{~mm}$ ) were observed in the aSDH group of rats in comparison with the control group in the superficial cortex (i.e., $<5 \mathrm{~mm}$ from the brain surface; Fig. 3D-F). The severities of reductions in regional brain blood flow and $\mathrm{P}_{b t} \mathrm{O}_{2}$ were related to the distance of the cerebral cortex from the surface.

\section{Pathological Examination Results: Diffuse Thrombosis of Capillaries, Pretreatment With Aspirin Prevents Thrombosis}

To understand the pathology of impaired microcirculation on the brain surface, we examined the changes in the small cerebral vessels and capillaries. As shown in Fig. 4, rats in the control group and the aSDH group were sacrificed for brain sectioning. H \& E staining showed diffuse thrombosis in cortical vessels and capillaries (Fig. 4A2), which was further confirmed by positive fibrin staining (Fig. 4B2). Strong albumin staining outside the vessels in the subcortical area (Fig. 4C2) indicated disruption of the blood-brain barrier (BBB), and this phenomenon of BBB breakdown was also supported by positive Evans blue staining (Fig. 4D). To further consolidate our findings, IHC staining was also performed. The IHC stains we chose were fibrin, GLUT1, and $\alpha$-SMA. GLUT1 is rich in endothelial cells, and $\alpha$-SMA is abundant in arterioles. The coexistence of these 2 fluorescent dyes showed yellowish light, which was the result of the mixed presence of fibrin plus GLUT1 or fibrin plus $\alpha$-SMA, both of which indicated intraluminal thrombosis. For fibrin staining alone, a background staining pattern was observed in the control (Fig. 5A) and the IP aspirin group (Fig. 5G), while areas of fibrin aggregation were found in the aSDH group (Fig. 5D). The yellowish light was evident in the aSDH group (with both GLUT1 and $\alpha$-SMA staining; Fig. $5 \mathrm{E}$ and $\mathrm{F}$ ) with $12.7 \%$ of thrombosis, but this was not observed in the control or the IP aspirin groups (as shown in Fig. 5B and $\mathrm{C}$ for the control group, and Fig. 5H and I for the IP aspirin group). Calculation of the area of thrombosis yielded a statistically significant difference between the control and SDH groups, as well as between the IP aspirin and SDH groups ( $\mathrm{p}<0.01$, Fig. 4J). Video 2 also demonstrates that pretreatment with aspirin prevented the stasis of blood flow, in contrast to Video 1.

VIDEO 2. Clip showing no obvious stasis of blood flow observed at 120 minutes after induction of aSDH when the rat had been pretreated with aspirin. Copyright Kuo-Chuan Wang. Published with permission. Click here to view.

\section{Discussion}

Acute SDH is traditionally defined as an SDH diagnosed within 3 days of TBI, despite some authors having extended this period to $1-2$ weeks. ${ }^{8}$ The nature and timing of neurosurgical intervention depends on multiple factors, including the patient's age, the location and size of the hematoma, and the medical and neurological condition of the patient. Surgical evacuation is often considered in patients with an aSDH thicker than $5 \mathrm{~mm}$ (as measured by axial CT scanning) and in those who have any signs of neurological deterioration, such as lethargy, change in mental status, or a focal neurological deficit. ${ }^{8}$ According to the consensus of most neurosurgeons, aSDH with a thickness less than $5 \mathrm{~mm}$ can be treated by the conservative 

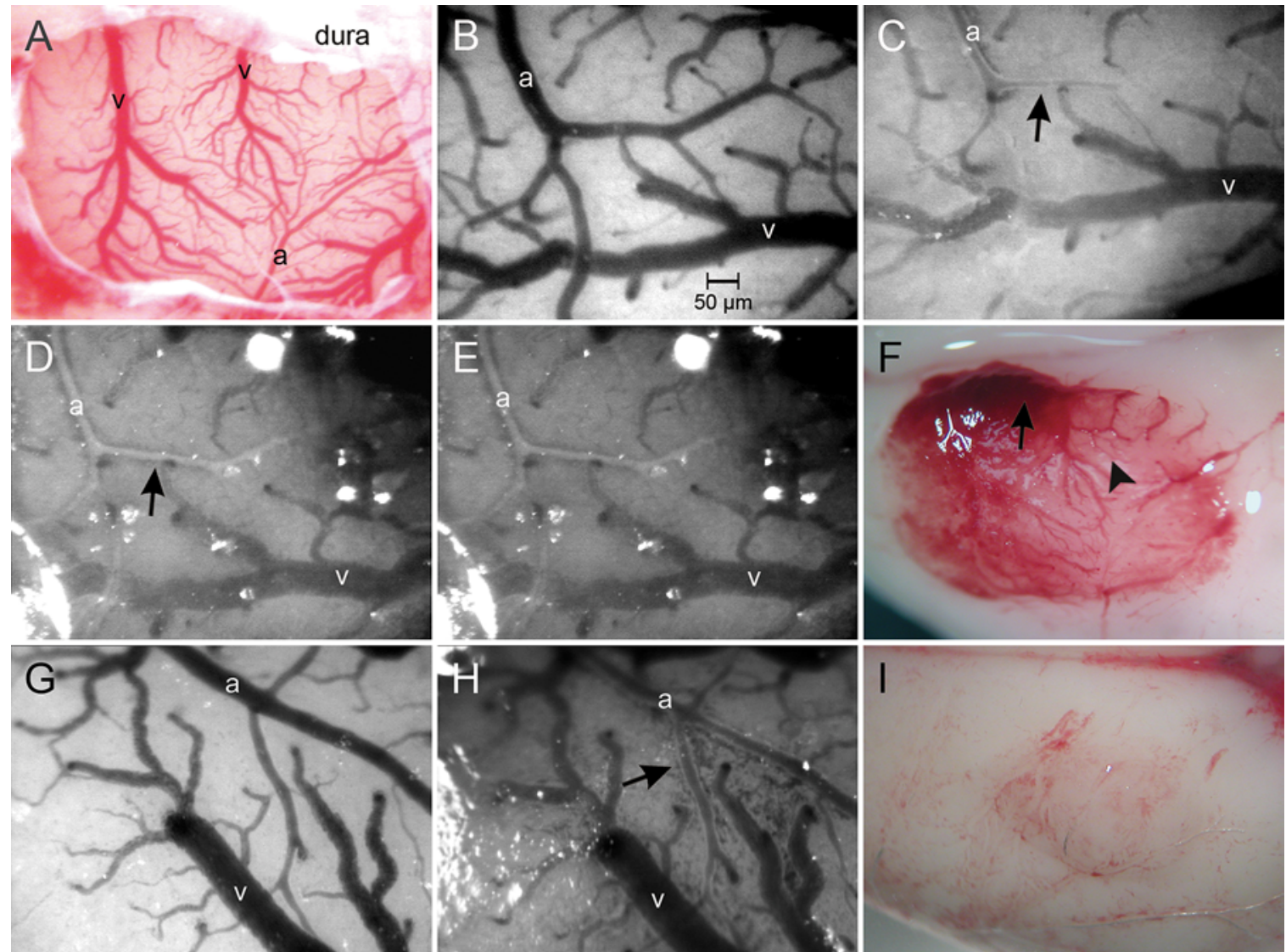

FIG. 2. Microcirculation impairment after experimental SDH. A $5 \times 5-\mathrm{mm}$ craniectomy window posterior to the frontal suture was created, the dura was opened using microscissors, and the capillary videoscope was aimed at the craniectomy window, which demonstrated significant diffuse vasoconstriction of the cortical vessels after superfusion of autologous blood onto the brain surface. A: Gross picture of the cortical microvasculature after opening of the dura under a dissecting microscope. The artery (a) and vein $(v)$ can be recognized by color, branching direction, and flow direction. B: Baseline vasculature before SDH under capillary videoscopy (bar $=50 \mu \mathrm{m})$. C: Instantaneous and marked vasospasm of the small cortical arteries (arrow) was observed 10 minutes after SDH. D: The cortical arteries (arrow) became thrombosed 120 minutes after SDH. E: Irrigation with normal saline after removal of the hematoma resulted in no obvious improvement of the thrombosed vessels. F: Gross picture of the brain surface after transcardiac perfusion with normal saline 120 minutes after SDH. Diffuse thrombosis of the cortical vessels (arrowhead) and distinct areas of necrosis (arrow) were observed. G-I: Images from an animal subject pretreated with aspirin. Instantaneous vasoconstriction still occurred after SDH; however, no thrombosis developed (arrow, H). There was no thrombosis of the cortical vessel in the aspirin-pretreated group after perfusion (I). Figure is available in color online only.

approach. However, we have observed that some patients with a small aSDH who receive conservative treatment present with progressive neurological deterioration or frequent seizures, and follow-up CT shows obvious brain edema underlying the hematoma without an obvious increase in the thickness of the hematoma. Although direct injury to the brain parenchyma itself from the initial impact cannot be excluded, the clinical progress still leads to doubts as to whether the subsequent changes may not be fully explained by a single traumatic event.

The mass effect of the hematoma, increased ICP, and acute brain swelling are all precipitating factors of ischemic brain damage, which might lead to poor outcomes after TBI. 1,6,33,35 Increased ICP in many circumstances reflects irreversible results of biochemical and cellular events that led to neuronal death, and therefore it is believed to be a result, rather than a cause, of the ischemic brain damage. ${ }^{1,6}$ Postmortem studies have demonstrated the presence of ischemic brain damage in two-thirds of deaths, whereas several studies in animal models have also shown ischemic brain damage after TBI. ${ }^{24,38}$ Ischemic damage is, therefore, one of the most important contributing factors of the sequelae of aSDH. Studies in rodents have shown that the affected underlying cerebral cortex is most susceptible, and the authors of these studies have suggested that direct local impact of the hematoma on microcirculation is most important. ${ }^{1,15,20,24}$ However, other investigators have demonstrated that remote areas in the territories of the anterior cerebral artery, the posterior cerebral artery, and the perforators are prone to sustain ischemic insult more frequently than the cortex underlying the site of the aSDH. ${ }^{1}$ In this novel animal model, we excluded the pressure effect of aSDH and demonstrated direct ischemic damage caused by the hematoma itself. The ischemic change extended to a certain depth from the cortex underneath the hematoma (up to $5 \mathrm{~mm}$ in the 

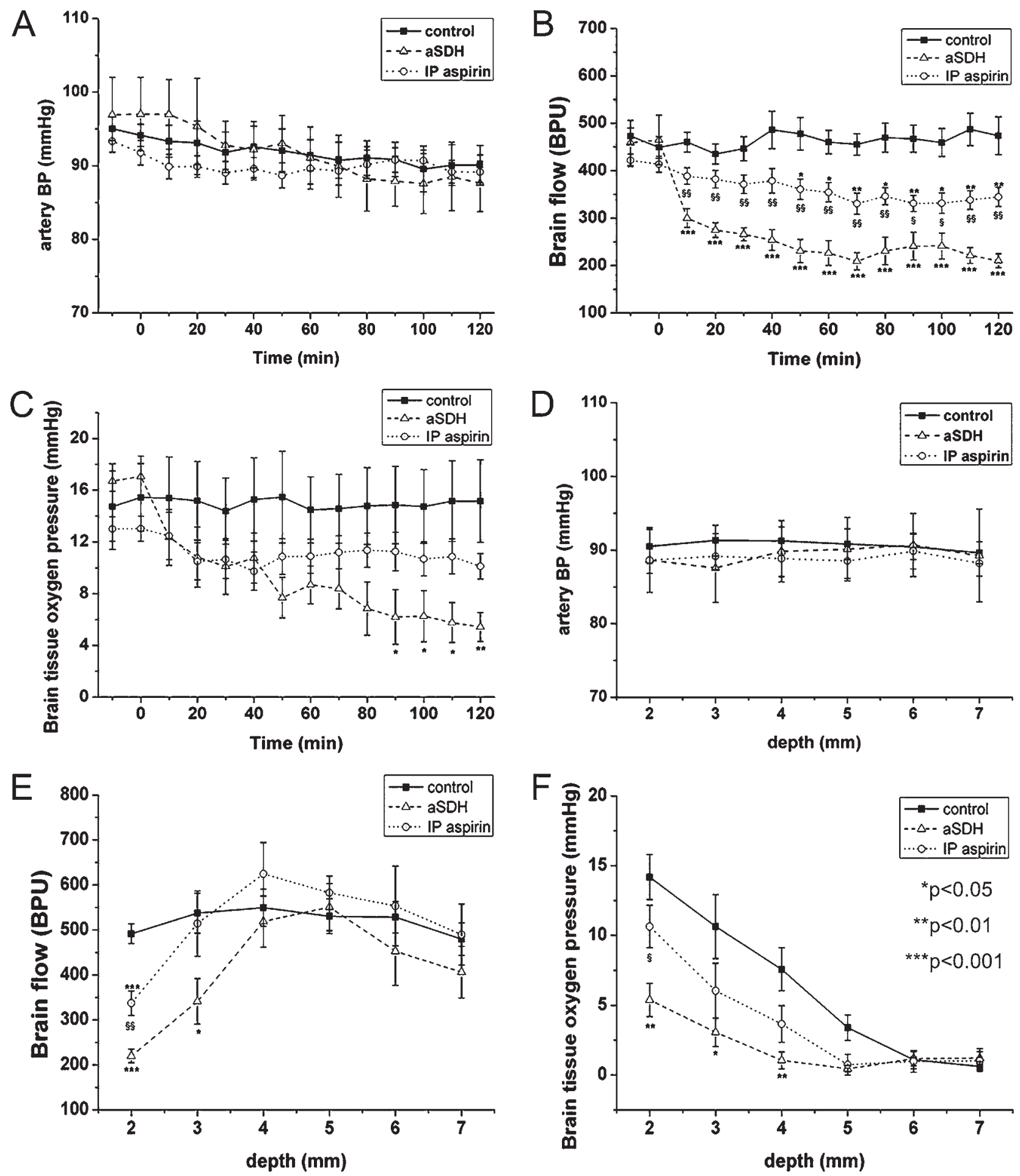

FIG. 3. Rapid decreases of regional brain blood flow and $\mathrm{P}_{b t} \mathrm{O}_{2}$ after experimental SDH. The animals were divided into 3 groups: the control group (control), the SDH without aspirin pretreatment group (aSDH), and the SDH with aspirin pretreatment group (IP aspirin). A: Regional blood flow and $\mathrm{P}_{\mathrm{bt}} \mathrm{O}_{2}$ values recorded at $2 \mathrm{~mm}$ in depth from the cortex demonstrated no difference in arterial blood pressure (BP) across groups. B: The SDH and IP aspirin groups both showed significant and rapid reduction of blood flow, but the degree of flow decrease was significantly lower in the IP aspirin group. C: The SDH group showed significant reduction in $\mathrm{P}_{\mathrm{bt}} \mathrm{O}_{2}$ after 90 minutes of $\mathrm{SDH}$ induction, while the IP aspirin group did not show a statistically significant drop in $\mathrm{P}_{\mathrm{bt}} \mathrm{O}_{2}$ as compared with the control group. D: Regional blood flow and $\mathrm{P}_{b t} \mathrm{O}_{2}$ values recorded at variable depths $(2-7 \mathrm{~mm})$ from 2 hours after $\mathrm{SDH}$ showed no significant difference in arterial BP across groups. E: There was a significant reduction of blood flow in the SDH group at 2 and $3 \mathrm{~mm}$, and significant reduction of blood flow in the IP aspirin group at $2 \mathrm{~mm}$ only; the reduction of blood flow was significantly less in the IP aspirin group than in the SDH group at a depth of $2 \mathrm{~mm}$. FIG. 3. (continued) $\rightarrow$ 
FIG. 3. F: A significant reduction of $\mathrm{P}_{\mathrm{bt}} \mathrm{O}_{2}$ was found in the SDH group from 2 to $4 \mathrm{~mm}$ in depth, while the IP aspirin group did not show a significant reduction of $\mathrm{P}_{b t} \mathrm{O}_{2}$. The data were analyzed using 1-way ANOVA, followed by Tukey's post hoc test for comparisons between groups. * Statistically significant difference between the control and SDH groups, as well as between the control and IP aspirin groups; $\S$ statistically significant difference between the SDH and IP aspirin groups. A single mark $=p<0.05,2$ marks $=p<0.01$, and 3 marks $=p<0.001$. BPU $=$ blood perfusion unit.

Wistar rat), which correlated with the subventricular zone (SVZ) that receives collateral circulation. This phenomenon can be explained both anatomically and by the development of cerebral blood supply during the embryonic stage. It has been well documented that cerebral cortical surfaces are rich in blood vessels but have no capillaries. ${ }^{29}$ The terminal arterioles turn at a right angle into the subcortical area and form a capillary plexus. It is known that there are 2 directions of cerebral angiogenesis during early embryonic development. After the initial extracerebral vascularization formed by migrated angioblasts from the splanchnopleuric mesoderm, the primitive vascular networks sprout deeper to grow the intracerebral vessels. Vascularization of the SVZ takes place at the earliest point by new branches growing around the ventricles, and these periventricular vessels originate from a basal vessel that grows into the SVZ. ${ }^{5}$ A second network of vasculature forms via an inside-out fashion to supply the developing white matter and the cortical plate at a later stage. The cortical branches are consequently located at specific cortical depths depending on the time of their formation, ${ }^{46}$ The deeper cerebral parenchyma (near the SVZ), by receiving 2 sets of vascular supply, is therefore more resistant to ischemic damage after aSDH.

Microcirculation refers to the delivery of oxygenated blood and nutrients to the smallest vasculature embedded within organ tissues. The arterioles responsible for microcirculation are surrounded by smooth muscle cells and are $10-100 \mu \mathrm{m}$ in diameter. ${ }^{40}$ Cerebral microcirculation is autoregulated under physiological conditions. Adequate perfusion is maintained within cells by means of vasoconstriction or vasodilation when the blood supplies are changed.$^{28}$ Previous reports have specifically discussed several microvascular changes in animal models of SAH: 1) microvascular constriction, 2) increased leukocyteendothelial cell interaction, 3) BBB breakdown, and 4) platelet aggregation and microthrombosis. ${ }^{18,44}$ All of these phenomena, with the exception of leukocyte-endothelial cell interaction, were observed in our experiments. These microvascular changes have been extensively discussed in SAH models, but much less attention has been given to aSDH; therefore, this may be our next target of study.

Previous studies on ischemic brain damage after TBI were commonly performed using rodent models of aSDH, which involved injecting blood into a closed subdural space. ${ }^{18,24,37}$ Using these animal models, researchers have successfully observed ischemic brain damage after TBI; however, it remains unclear as to whether the ischemic change resulted from direct compression or from the toxic effect of the hematoma. Duhaime et al. ${ }^{12}$ demonstrated that blood on the cortical surface without pressure is insufficient as a cause of underlying infarction, while Kuroda and Bullock ${ }^{20}$ demonstrated that enlargement of the ischemic tissue zone occurs after SDH, a process accompanied by transient glucose hypermetabolism and a brief increase in extracellular glutamate in the ischemic cortex..$^{22}$ This excitotoxic process originates from the initial ischemic core and spreads out to nearby brain tissue; it cannot be reversed after hematoma removal., ${ }^{9,20,22}$ Other studies have concluded that traumatic and ischemic brain injuries may share a number of common mechanisms of injury, such as free radical production and excitotoxicity. $3,10,11,13,17,21,27,45$ Taking these results together, it is still not clear as to whether increased ICP, vasoactive effects, or toxicity of the blood itself is responsible for triggering or maintaining these ischemic processes. ${ }^{12}$ We did not study the metabolism in our experiment; however, the evidence of significant thrombosis in the absence of increased ICP emphasizes the need to obtain further information about the roles of excitotoxic and vasoactive processes in future studies.

To test the antithrombotic effect of aspirin in this model, we chose a dose of $150 \mathrm{mg} / \mathrm{kg}$ administered via the IP route 1 hour prior to craniectomy. A high dose is required to ensure an adequate drug serum level over the time period from aSDH induction until sacrifice of the animal. Furthermore, this high dose is mandatory because of the high drug clearance rate in this species. The median effective dose for analgesic and antiinflammatory effects ranges between 150 and $350 \mathrm{mg} / \mathrm{kg}{ }^{26,39}$ According to the interspecies scaling relationship formula for aspirin, ${ }^{25}$ this $150 \mathrm{mg} / \mathrm{kg}$ should be equivalent to $1000 \mathrm{mg}$ in a $70-\mathrm{kg}$ human, which is a clinically applicable dose. ${ }^{7}$ We demonstrated that pretreatment with aspirin prevented thrombosis of cortical vessels underneath the hematoma. However, in clinical situations, aspirin may be contraindicated in patients with acute head injury. Further study is needed to clarify the role of aspirin in the clinical setting during aSDH and the appropriate timing of its application.

There were some limitations in this study. First, irrigation of normal saline in the animal model resulted in improvement of microcirculation, but whether this irrigation can improve neurological deficit is as yet unknown. We do not know at which time point the thrombosed vessels will eventually become irreversibly obliterated. Another drawback was that this animal model is only suitable for short-term study, as prolonged anesthesia may affect the hemodynamics of the animal. Aspirin appeared to be effective in decreasing the thrombosis, but the timing and indication of usage in the clinical setting are unclear. Nevertheless, the results of this experimental animal model provide us with alternative viewpoints regarding the treatment of aSDH, other than simply aiming for management of the ICP.

\section{Conclusions}

We presented the cases of 2 patients with small aSDHs who suffered from repeated seizures and progressive neurological deficits. Based on the intraoperative findings, we 

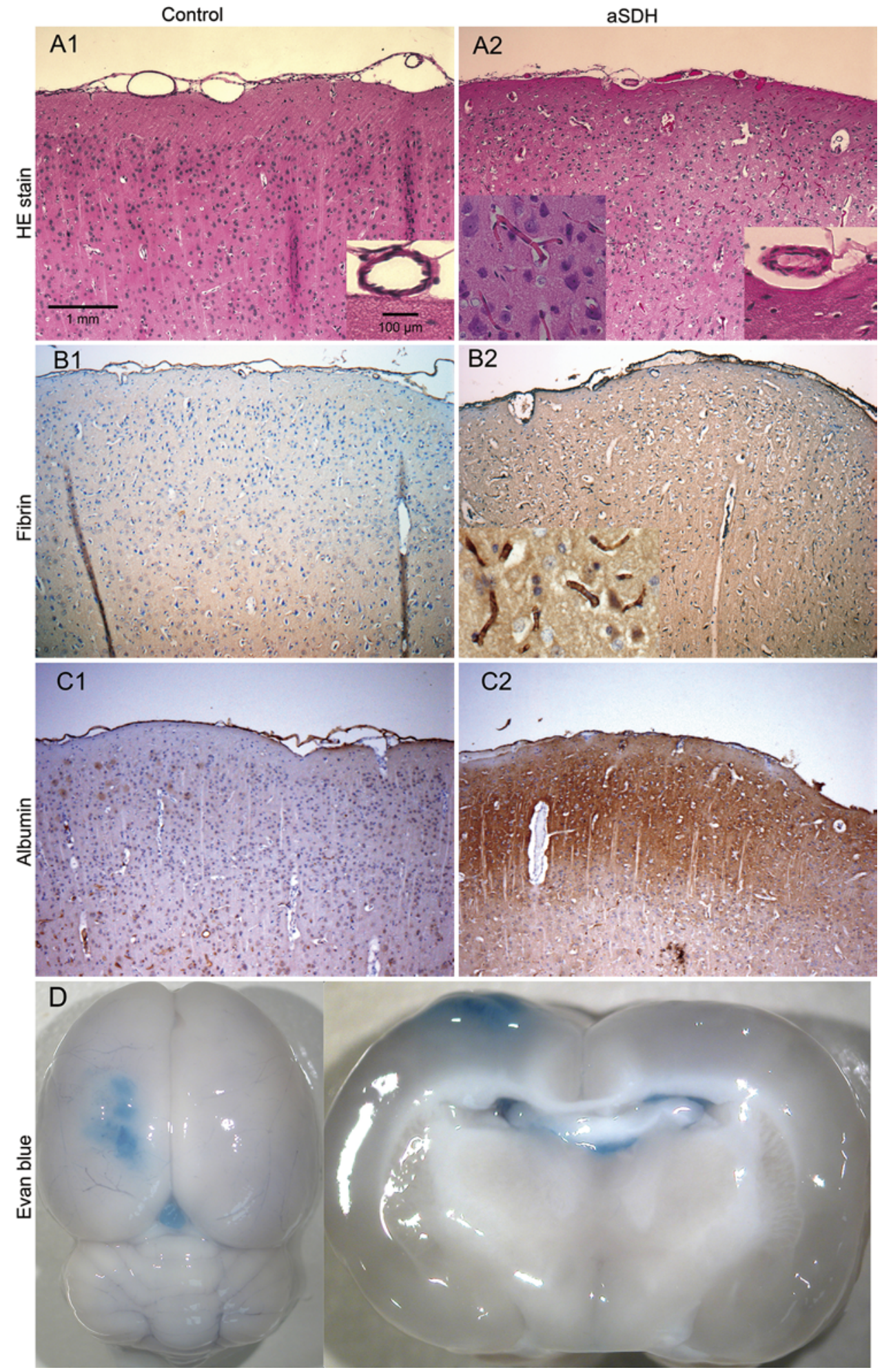

FIG. 4. Pathological examination of rat brains in the control group (A1, B1, and C1) and 4 hours after experimental aSDH (A2, $B 2$, and $\mathbf{C 2}$ ). Diffuse thrombosis of cortical vessels and capillaries beneath the cortex was demonstrated by $H$ \& $E$ staining (A2). Positive staining of fibrin confirmed thrombi formation within the vessels of the subcortical regions (B2). Disruption of the BBB was demonstrated by strong albumin staining outside the vessels in the subcortical area (C2), and was also supported by positive Evans blue staining (D; only an SDH group rat is shown). Figure is available in color online only. 


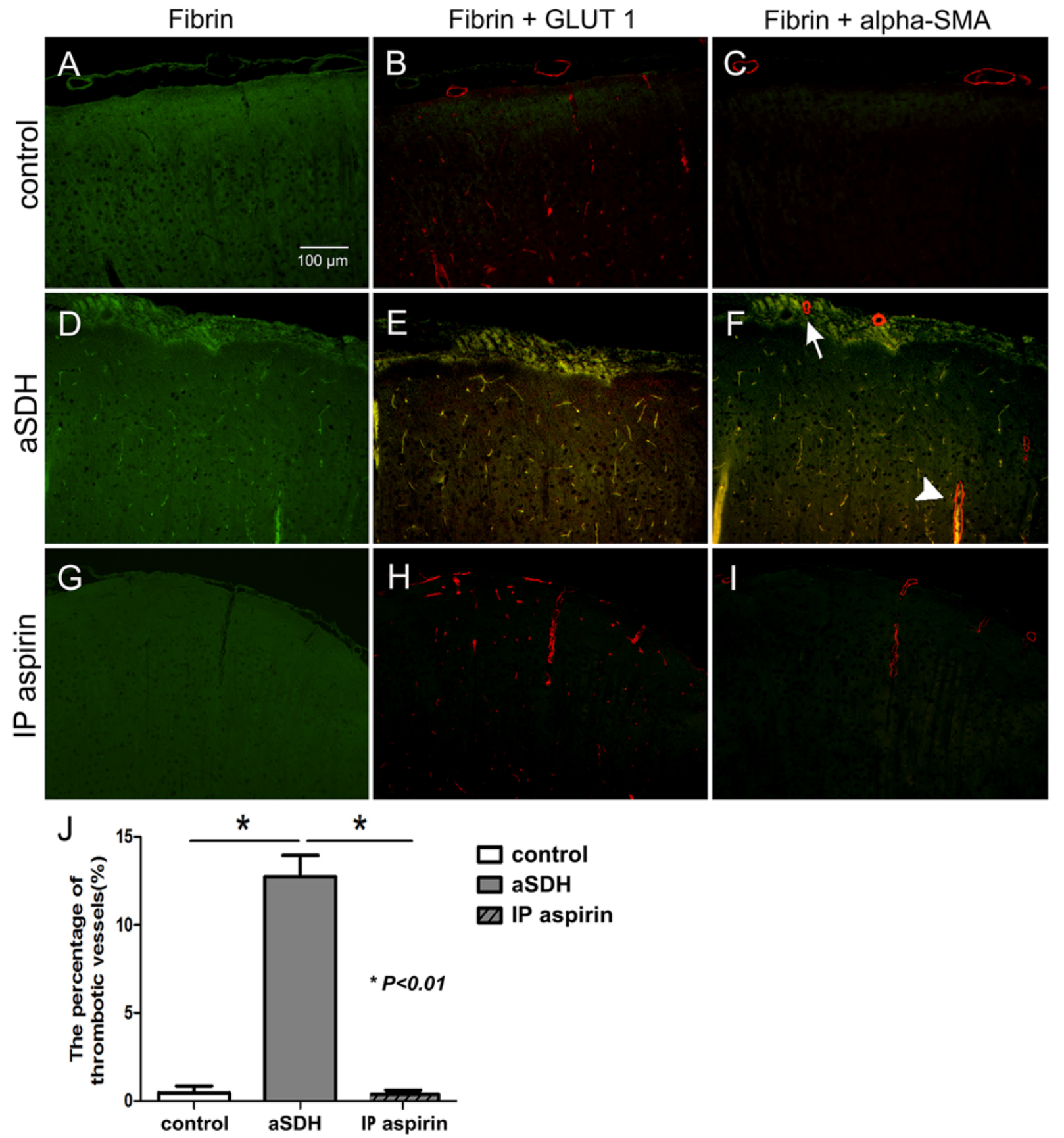

FIG. 5. Microthrombosis in the rat brain after experimental aSDH. Animals were divided into the control group (control), the aSDH without aspirin pretreatment group (SDH), and the aSDH with aspirin pretreatment group (IP aspirin). IHC staining including fibrin (green in fluorescence), GLUT1 (red in fluorescence), and $\alpha$-SMA (also red in fluorescence) was performed in each group. Fibrin staining in the control group revealed some background staining pattern (A). The endothelium of arterioles, venules, and capillaries was observed by GLUT1 staining (B), but only the arterioles were positively stained by $\alpha$-SMA (C). In the aSDH group, areas of dense fibrin aggregation could be seen (D), and these aggregations were actually thrombi in the capillaries of the brain parenchyma (E); these were absent in the control group. The arterioles on the brain surface (arrow) showed vasoconstriction with a thickened arterial wall and small lumen $(\mathbf{F})$. There were also thrombi in the perpendicular arterioles (arrowhead in panel $F$ ). The IP aspirin group showed no obvious areas of dense fibrin aggregation $(\mathbf{G})$, and less thrombi $(\mathbf{H})$ and vasoconstriction (I). The proportion of fibrin-positive capillaries was quantitatively analyzed $(\mathbf{J})$. Figure is available in color online only.

set up a new animal model and proved that the hematoma itself could cause microcirculation impairment of the brain cortex, and may be responsible for the neurological deterioration. Direct visualization of the cerebral micro- circulation by capillary videoscopy assisted in the identification of microcirculation impairment after aSDH. Measurements of regional blood flow and $\mathrm{P}_{\mathrm{bt}} \mathrm{O}_{2}$ further demonstrated ischemic cerebral damage at different depths from 
the cortex. Furthermore, pretreatment with aspirin was proven to be capable of reducing ischemic damage after aSDH, but further investigation is still needed to elucidate its effects.

\section{Acknowledgments}

We are grateful for the support of the 3rd Core Facility at National Taiwan University Hospital. This study received research grants from National Taiwan University Hospital (no. NTUH.102-M2313) and the National Science Council, Taiwan (no. 103-2314-B-002-118-MY2).

\section{References}

1. Abe M, Udono H, Tabuchi K, Uchino A, Yoshikai T, Taki K: Analysis of ischemic brain damage in cases of acute subdural hematomas. Surg Neurol 59:464-472, 2003

2. Adams JH, Graham DI, Gennarelli TA: Head injury in man and experimental animals: neuropathology. Acta Neurochir Suppl (Wien) 32:15-30, 1983

3. Arundine M, Tymianski M: Molecular mechanisms of glutamate-dependent neurodegeneration in ischemia and traumatic brain injury. Cell Mol Life Sci 61:657-668, 2004

4. Baechli H, Behzad M, Schreckenberger M, Buchholz HG, Heimann A, Kempski O, et al: Blood constituents trigger brain swelling, tissue death, and reduction of glucose metabolism early after acute subdural hematoma in rats. J Cereb Blood Flow Metab 30:576-585, 2010

5. Bär T: The Vascular System of the Cerebral Cortex. Berlin: Springer, 1980

6. Bouma GJ, Muizelaar JP, Choi SC, Newlon PG, Young HF: Cerebral circulation and metabolism after severe traumatic brain injury: the elusive role of ischemia. J Neurosurg 75:685-693, 1991

7. Burke A, Smyth EM, Fitzgerald GA: Analgesic-antipyretic agents; pharmacotherapy of gout, in Brunton LL, Lazo JS, Parker KL (eds): Goodman \& Gilman's The Pharmacological Basis of Therapeutics, ed 11. New York: McGraw-Hill, 2006, pp 671-715

8. Bullock MR, Chesnut R, Ghajar J, Gordon D, Hartl R, Newell DW, et al: Surgical management of acute subdural hematomas. Neurosurgery 58 (3 Suppl):S16-S24, Si-Siv, 2006

9. Bullock R, Butcher SP, Chen MH, Kendall L, McCulloch J: Correlation of the extracellular glutamate concentration with extent of blood flow reduction after subdural hematoma in the rat. J Neurosurg 74:794-802, 1991

10. Chan PH, Epstein CJ, Li Y, Huang TT, Carlson E, Kinouchi $\mathrm{H}$, et al: Transgenic mice and knockout mutants in the study of oxidative stress in brain injury. J Neurotrauma 12:815824,1995

11. Chen MH, Bullock R, Graham DI, Miller JD, McCulloch J: Ischemic neuronal damage after acute subdural hematoma in the rat: effects of pretreatment with a glutamate antagonist. J Neurosurg 74:944-950, 1991

12. Duhaime AC, Gennarelli LM, Yachnis A: Acute subdural hematoma: is the blood itself toxic? J Neurotrauma 11:669678, 1994

13. Faden AI, Demediuk P, Panter SS, Vink R: The role of excitatory amino acids and NMDA receptors in traumatic brain injury. Science 244:798-800, 1989

14. Fell DA, Fitzgerald S, Moiel RH, Caram P: Acute subdural hematomas. Review of 144 cases. J Neurosurg 42:37-42, 1975

15. Fujisawa H, Maxwell WL, Graham DI, Reasdale GM, Bullock R: Focal microvascular occlusion after acute subdural haematoma in the rat: a mechanism for ischaemic damage and brain swelling? Acta Neurochir Suppl (Wien) 60:193196, 1994

16. Hyder AA, Wunderlich CA, Puvanachandra P, Gururaj G,
Kobusingye OC: The impact of traumatic brain injuries: a global perspective. NeuroRehabilitation 22:341-353, 2007

17. Ikeda Y, Long DM: The molecular basis of brain injury and brain edema: the role of oxygen free radicals. Neurosurgery 27:1-11, 1990

18. Karabiyikoglu M, Keep R, Hua Y, Xi G: Acute subdural hematoma: new model delineation and effects of coagulation inhibitors. Neurosurgery 57:565-572, 2005

19. Koç RK, Akdemir H, Oktem IS, Meral M, Menkü A: Acute subdural hematoma: outcome and outcome prediction. Neurosurg Rev 20:239-244, 1997

20. Kuroda Y, Bullock R: Local cerebral blood flow mapping before and after removal of acute subdural hematoma in the rat. Neurosurgery 30:687-691, 1992

21. Kuroda Y, Fujisawa H, Strebel S, Graham DI, Bullock R: Effect of neuroprotective N-methyl-D-aspartate antagonists on increased intracranial pressure: studies in the rat acute subdural hematoma model. Neurosurgery 35:106-112, 1994

22. Kuroda Y, Inglis FM, Miller JD, McCulloch J, Graham DI, Bullock R: Transient glucose hypermetabolism after acute subdural hematoma in the rat. J Neurosurg 76:471-477, 1992

23. Leitgeb J, Mauritz W, Brazinova A, Janciak I, Majdan M, Wilbacher I, et al: Outcome after severe brain trauma due to acute subdural hematoma. J Neurosurg 117:324-333, 2012

24. Miller JD, Bullock R, Graham DI, Chen MH, Teasdale GM: Ischemic brain damage in a model of acute subdural hematoma. Neurosurgery 27:433-439, 1990

25. Mordentia J, Chappell W: The use of interspecies scaling in toxicokinetics, in Yacobi A, Skelly JP, Batra VK (eds): Toxicokinetics and New Drug Development. New York: Pergamon Press, 1989, pp 42-96

26. Muller DN, Heissmeyer V, Dechend R, Hampich F, Park JK, Fiebeler A, et al: Aspirin inhibits NF-KB and protects from angiotensin II-induced organ damage. FASEB J 15:18221824,2001

27. Myseros JS, Bullock R: The rationale for glutamate antagonists in the treatment of traumatic brain injury. Ann N Y Acad Sci 765:262-271, 298, 1995

28. Niimi H, Komai Y, Yamaguchi S: Blood flow regulation in the cerebral microvasculature with an arcadal network: a numerical simulation. Indian J Exp Biol 45:41-47, 2007

29. Nonaka H, Akima M, Nagayama T, Hatori T, Zhang Z, Ihara F: Microvasculature of the human cerebral meninges. Neuropathology 23:129-135, 2003

30. Otite F, Mink S, Tan CO, Puri A, Zamani AA, Mehregan A, et al: Impaired cerebral autoregulation is associated with vasospasm and delayed cerebral ischemia in subarachnoid hemorrhage. Stroke 45:677-682, 2014

31. Patel TR, Schielke GP, Hoff JT, Keep RF, Lorris Betz A: Comparison of cerebral blood flow and injury following intracerebral and subdural hematoma in the rat. Brain Res 829:125-133, 1999

32. Prabhakaran $S$, Naidech AM: Ischemic brain injury after intracerebral hemorrhage: a critical review. Stroke 43:22582263, 2012

33. Ropper AH: Lateral displacement of the brain and level of consciousness in patients with an acute hemispheral mass. $\mathbf{N}$ Engl J Med 314:953-958, 1986

34. Rosen $\varnothing \mathbf{r n}$ J, Gjerris F: Long-term follow-up review of patients with acute and subacute subdural hematomas. J Neurosurg 48:345-349, 1978

35. Ross DA, Olsen WL, Ross AM, Andrews BT, Pitts LH: Brain shift, level of consciousness, and restoration of consciousness in patients with acute intracranial hematoma. J Neurosurg 71:498-502, 1989

36. Ryan CG, Thompson RE, Temkin NR, Crane PK, Ellenbogen 
RG, Elmore JG: Acute traumatic subdural hematoma: current mortality and functional outcomes in adult patients at a Level I trauma center. J Trauma Acute Care Surg 73:1348-1354, 2012

37. Sasaki M, Dunn L: A model of acute subdural hematoma in the mouse. J Neurotrauma 18:1241-1246, 2001

38. Schröder ML, Muizelaar JP, Kuta AJ: Documented reversal of global ischemia immediately after removal of an acute subdural hematoma. Report of two cases. J Neurosurg 80:324-327, 1994

39. Schrör K: Pharmacology, in Acetylsalicylic Acid. Weinheim, Germany: Wiley-Blackwell, 2009, pp 33-128

40. Sehba FA, Friedrich V: Early micro vascular changes after subarachnoid hemorrhage. Acta Neurochir Suppl 110:49_ 55,2011

41. Song JN, Chen H, Zhang M, Zhao YL, Ma XD: Dynamic change in cerebral microcirculation and focal cerebral metabolism in experimental subarachnoid hemorrhage in rabbits. Metab Brain Dis 28:33-43, 2013

42. Tallon JM, Ackroyd-Stolarz S, Karim SA, Clarke DB: The epidemiology of surgically treated acute subdural and epidural hematomas in patients with head injuries: a populationbased study. Can J Surg 51:339-345, 2008

43. Tian HL, Chen SW, Xu T, Hu J, Rong BY, Wang G, et al: Risk factors related to hospital mortality in patients with isolated traumatic acute subdural haematoma: analysis of 308 patients undergone surgery. Chin Med J (Engl) 121:10801084,2008

44. Tso MK, Macdonald RL: Acute microvascular changes after subarachnoid hemorrhage and transient global cerebral ischemia. Stroke Res Treat 2013:425281, 2013

45. Tsuchida E, Bullock R: The effect of the glycine site-specific $\mathrm{N}$-methyl-D-aspartate antagonist ACEA1021 on ischemic brain damage caused by acute subdural hematoma in the rat. J Neurotrauma 12:279-288, 1995
46. Wittko-Schneider IM, Schneider FT, Plate KH: Cerebral angiogenesis during development: Who is conducting the orchestra? in Milner R (ed): Cerebral Angiogenesis: Methods and Protocols. New York: Springer, 2014, pp 3-20

\section{Disclosures}

The authors report no conflict of interest concerning the materials or methods used in this study or the findings specified in this paper.

\section{Author Contributions}

Conception and design: KC Wang. Acquisition of data: KC Wang, Lee, Lin. Analysis and interpretation of data: KC Wang, Lee, APH Huang. Drafting the article: HC Wang. Critically revising the article: KC Wang, Tsai, SJ Huang, Hsieh. Reviewed submitted version of manuscript: KC Wang, HC Wang, Tsai. Approved the final version of the manuscript on behalf of all authors: KC Wang. Statistical analysis: KC Wang. Study supervision: KC Wang.

\section{Supplemental Information Videos}

Video 1. https://vimeo.com/228067664.

Video 2. https://vimeo.com/228067782.

\section{Correspondence}

Kuo-Chuan Wang, Division of Neurosurgery, Department of Surgery, National Taiwan University Hospital, No. 7, Chung Shan S Rd., Zhongzheng Dist., Taipei 10002, Taiwan. email: wang081466@yahoo.com.tw. 